SUDRÉ CP; CRUZ CD; RODRIGUES R; RIVA EM; AMARAL JÚNIOR AT; SILVA DJH; PEREIRA TNS. 2006. Variáveis multicategóricas na determinação da divergência genética entre acessos de pimenta e pimentão. Horticultura Brasileira, 24: 88-93.

\title{
Variáveis multicategóricas na determinação da divergência genética entre acessos de pimenta e pimentão
}

\author{
Cláudia P Sudréé1; Cosme Damião Cruz ${ }^{2 /}$; Rosana Rodrigues ${ }^{1 /}$; Elaine M Riva ${ }^{1 /}$; Antônio T do Amaral \\ Júnior ${ }^{1 /}$; Derly José H da Silva ${ }^{2 /}$; Telma Nair S Pereira ${ }^{1 /}$ \\ ${ }^{1}$ UENF, Av. Alberto Lamego, 2000, Parque Califórnia, 28013-602 Campos dos Goytacazes-RJ; E-mail: cpombo@uenf.br; ${ }^{2 / U F V, ~ D e p ~}{ }^{\text {to }}$ \\ Fitotecnia, 36571-000 Viçosa-MG
}

\section{RESUMO}

A manutenção de acessos de Capsicum em bancos de germoplasma tem crescido em importância para preservação da diversidade e uso em programas de melhoramento genético. Este trabalho visou avaliar a divergência genética entre acessos de Capsicum spp. e verificar a eficiência da utilização de medidas de dissimilaridade e da discriminação de genótipos em espécies, baseando-se em 13 variáveis multicategóricas. O experimento foi realizado em Campos dos Goytacazes, RJ, em blocos ao acaso com três repetições utilizando 59 acessos com dez plantas por parcela, entre novembro/2001 e julho/2002. Os descritores considerados foram: cor do caule, da antera e da corola; número de flores por axila; posição da flor; hábito de crescimento; cor do fruto imaturo e maduro; forma e epiderme do fruto; cor da semente e da folha cotiledonar; número de lóculos; constrição anular do cálice e do fruto. Os dados foram obtidos pela moda de cada descritor seguindo-se os critérios do IPGRI. As análises realizadas foram: agrupamento pelo método de Tocher e Hierárquico do Vizinho Mais Próximo; Projeção das Distâncias no Plano e Análise Discriminante de Anderson. O método de Tocher diferenciou as espécies de $C$. annuum var. annuum, $C$. annuum var. glabriusculum, C. chinense e C. pubescens, confirmando que os descritores qualitativos essenciais para a caracterização de Capsicum spp. devem ser utilizados no manejo de bancos de germoplasma. Os métodos de Tocher, Hierárquico do Vizinho Mais Próximo e Projeção das Distâncias no Plano foram concordantes e eficazes em agrupar acessos com pequena distância genética entre si. As funções discriminantes de Anderson identificaram 100\% das espécies e variedades botânicas estudadas.

Palavras-chave: Capsicum spp; banco de germoplasma; análise multivariada.

\begin{abstract}
Multicategoric variables for determining the genetic divergence among sweet and chilli peppers
\end{abstract}

Accessions of Capsicum maintained in germplasm banks are important not only for diversity preservation but also for plant breeding purposes. In this work the genetic divergence among Capsicum spp. accessions, were studied to verify the efficiency of dissimilarity measures and to discriminate genotypes in species, based on 13 multicategoric variables. The experiment was conducted in Campos dos Goytacazes, RJ, in randomized blocks with three replications testing 59 accessions, ten plants per plot, from November, 2001 to July, 2002. The following descriptors were considered: cotyledoneus leaf color; stem color, anther color; corolla color; number of flowers per axil; flower position; plant growth habit; fruit color at intermediate stage; fruit color at mature stage; fruit shape; fruit surface; seed color; number of locules; calyx annular constriction, and neck at base of fruit. The data were collected using mode of each descriptor following the IPGRI (1995) criteria. The data were analyzed according to Tocher optimization method, Nearest Neighbor, distance plotted in plain and discriminant analysis. Qualitative traits analyzed by Tocher's method were efficient to differentiate among the species $C$. annuum var. annuиm, C. annuиm var. glabriusculum, $C$. chinense, and $C$. pubescens. This result confirms that qualitative descriptors considered essential for morphological and agronomic characterization must be used in germplasm bank management. Tocher's method and Nearest Neighbor methods and distance plotted in plain showed resemblance and were efficient in clustering accessions with small genetic distance among them. Using functions of Anderson's discriminant analysis it was possible to identify $100 \%$ of species and botanic variety used in this study.

Keywords: Capsicum spp; germplasm bank; multivariate analysis.

\section{(Recebido para publicação em 31 de março de 2005; aceito em 27 de setembro de 2005)}

\begin{abstract}
A determinação da divergência genética existente em bancos de germoplasma permite, entre outros, a caracterização de acessos, a identificação de duplicatas (Coimbra et al., 2001) e a identificação de genitores adequados à obtenção de híbridos com maior efeito heterótico potencial, para serem utilizados em programas de melhoramento (Cruz \& Carneiro, 2003).

Com o intuito de obter cultivares comerciais com características exigidas
\end{abstract}

pelos produtores e consumidores, o melhorista deve estar atento às características que sejam importantes para a qualidade do produto comercializado como forma, coloração, e tamanho do fruto (Cruz \& Carneiro, 2003). Para o gênero Capsicum, caracteres qualitativos como formato do fruto, cor do fruto imaturo e maduro, textura da epiderme, são alguns dos caracteres importantes para o mercado consumidor. Os pimentões são mais comercializados na for- ma imatura (verde). Entretanto, ocorre demanda por frutos maduros (vermelhos, amarelos, laranjas, cremes e até roxos) (Filgueira, 2000). Há regiões que preferem o fruto do tipo "quadrado" enquanto outras dão preferência ao tipo "cônico". Cultivares com frutos de formato quadrado, como 'Yolo Wonder' são pouco plantadas no Centro-Sul, porém preferidos nas regiões Nordeste e Sul. Nas regiões Sudeste e Centro-Oeste, prevalecem as cultivares com frutos 
de formato alongado (cônico ou piramidal). Em se tratando de pimentas, as mais consumidas na Região Nordeste são maduras, com frutos pequenos e redondos (conhecidos popularmente como "pimenta-de-bode"); enquanto as que produzem frutos finos e alongados (conhecidos como "pimenta malagueta") são as mais consumidas na Região Sudeste.

Caracteres qualitativos também são utilizados nas chaves de identificação taxonômicas de famílias, gêneros e espécies vegetais. Para Capsicum spp., Sudré (2003) verificou correlação (Spearman) superior a 0,90 entre cor da flor e espécie, ratificando a importância de se determinar dados qualitativos na caracterização de banco de germoplasma.

Normalmente, os dados qualitativos utilizados nas análises multivariadas são de natureza binária, isto é, ausência (0) ou presença (1) de uma determinada característica, porém para a caracterização morfoagronômica são mais utilizadas as variáveis multicategóricas, com mais de duas classes por variável. É possível transformar dados multicategóricos em binários, por exemplo, para o descritor forma do fruto: alongado (1) e não alongado (0); redondo (1) ou não redondo (0); triangular (1) e não triangular (0) e assim sucessivamente até se verificar a presença ou ausência de todas as formas de fruto possíveis para a cultura estudada (Liberato, 1995). Ao proceder esta transformação há perda de informações, acarretando em menor precisão nas estimativas de dissimilaridade entre acessos (Cruz \& Carneiro, 2003).

Para variáveis multicategóricas usase o índice de similaridade, em que determinado valor expressa a porcentagem de coincidência de similaridade considerando os vários caracteres analisados. Assim, obtém-se a matriz de dissimilaridade a partir desses índices para posterior realização das análises de agrupamento (Coimbra et al., 2001; Cruz \& Carneiro, 2003).

Coimbra et al. (2001), estudando a divergência genética entre 16 genótipos de milho em relação a sete descritores qualitativos, concluíram que a análise de agrupamento utilizando-se matrizes de dissimilaridade obtidas a partir de dados multicategóricos consiste em alternativa viável para se avaliar divergência entre genótipos, sendo constatada divergência entre os genótipos avaliados.

Abreu et al. (2002) utilizaram 33 variáveis multicategóricas para estudar a divergência genética entre 34 acessos de tomateiro. A análise permitiu a separação dos acessos em dez grupos pelo método de agrupamento de Tocher, e as funções discriminantes estimadas foram eficazes em alocar os genótipos em suas devidas classes.

Para a análise discriminante de Anderson (1958) é necessário que, previamente, os acessos estejam divididos em populações ou grupos distintos. Com estas informações, geram-se funções, que são combinações lineares das características avaliadas, tendo por finalidade obter a melhor discriminação entre os indivíduos, alocando-os em seus devidos grupos ou populações. Estas funções passam a ser de grande valia por permitirem classificar novos genótipos, de comportamento desconhecido, nas populações já conhecidas. A eficácia das variáveis utilizadas em promover a discriminação também é avaliada, permitindo conhecer a adequação da função estimada.

Assis (2001) empregou a técnica de análise discriminante em 301 acessos de Brachiaria spp. As funções discriminantes foram obtidas a partir de 24 variáveis para cada espécie, e ao reclassificar os acessos utilizando estas funções, 37 dos 301 indivíduos alocaram-se em grupos aos quais não pertenciam, sendo a taxa de erro aparente igual a $12,29 \%$. Neste trabalho, concluiu-se que a utilização das análises discriminantes foi eficaz na discriminação das espécies.

Abreu (2001) obteve funções discriminantes eficientes em alocar genótipos de feijão-vagem (Phaseolus vulgaris L.) em duas classes: vagens do tipo "macarrão" (redondo) e "manteiga" (chato).

Este trabalho teve como objetivos avaliar a divergência genética entre acessos de Capsicum spp., verificar a eficiência da utilização de medidas de dissimilaridade e analisar a eficiência de discriminação de genótipos em suas respectivas classes (espécies), baseando-se em variáveis multicategóricas.

\section{MATERIAL E MÉTODOS}

Cinqüenta e nove acessos da Coleção de germoplasma de Capsicum spp. da UENF, provenientes de diversas regiões geográficas, foram utilizados neste estudo (Tabela 1). O experimento foi realizado na Unidade de Apoio à Pesquisa da UENF, utilizando-se o delineamento de blocos ao acaso com três repetições, 59 tratamentos (acessos) com dez plantas por parcela, de novembro/ 2001 a julho/2002. Outras informações sobre os acessos estudados e as condições experimentais podem ser consultadas em Sudré et al. (2005) e Sudré (2003).

Os dados foram obtidos por meio da moda de cada descritor, num total de 30 plantas. Foram estudadas 13 variáveis multicategóricas e duas binárias, consideradas descritores essenciais pelo International Plant Genetic Resource Institute (IPGRI, 1995), quais sejam: cor do caule; cor da antera; cor da corola; número de flores por axila; posição da flor; hábito de crescimento; cor do fruto no estádio intermediário; cor do fruto no estádio maduro; forma do fruto; epiderme do fruto; cor da semente; número de lóculos; cor da folha cotiledonar; constrição anular do cálice e presença de constrição do fruto. Os dados para cada variável foram tomados seguindo-se os critérios propostos pelo IPGRI (1995).

A análise estatística consistiu na obtenção da matriz de dissimilaridades com variáveis multicategóricas. Para obtenção da matriz de dissimilaridade foi utilizada a moda de cada variável por acesso, sem repetição. Com as medidas de dissimilaridade e com auxílio do programa GENES (Cruz, 2001) versão 2004, foram realizadas as análises: agrupamento pelo Método de Tocher e o Método Hierárquico do Vizinho Mais Próximo (VMP); Projeção das Distâncias no Plano e Análise Discriminante de Anderson (1958). 
Tabela 1. Lista dos acessos de Capsicum spp. da coleção de germoplasma do CCTA/UENF utilizados como tratamento. Campos dos Goytacazes, UENF, 2002.

\begin{tabular}{|c|c|c|}
\hline Acesso $\mathrm{N}^{\circ}$ & UENF $\mathbf{N}^{\circ}$ & Espécie \\
\hline 02 & 1381 & C. annuum var. annuum \\
\hline 03 & 1382 & C. annuum var. annuum \\
\hline 04 & 1417 & C. baccatum var. pendulum \\
\hline 05 & 1418 & C. chinense Jacq. \\
\hline 06 & 1419 & C. chinense Jacq. \\
\hline 07 & 1420 & C. annuum var. annuum \\
\hline 08 & 1421 & C. annuum var. annuum \\
\hline 09 & 1422 & C. annuum var. annuum \\
\hline 10 & 1423 & C. annuum var. annuum \\
\hline 11 & 1424 & C. chinense Jacq. \\
\hline 12 & 1425 & C. frutescens L. \\
\hline 13 & 1426 & C. baccatum var. pendulum \\
\hline 14 & 1489 & C. baccatum var. pendulum \\
\hline 15 & 1490 & C. baccatum var. pendulum \\
\hline 16 & 1491 & C. frutescens $\mathrm{L}$. \\
\hline 17 & 1492 & C. baccatum var. pendulum \\
\hline 18 & 1493 & C. chinense Jacq. \\
\hline 19 & 1494 & C. baccatum var. pendulum \\
\hline 20 & 1495 & C. baccatum var. baccatum \\
\hline 21 & 1496 & C. baccatum var. pendulum \\
\hline 22 & 1497 & C. chinense Jacq. \\
\hline 23 & 1498 & C. chinense Jacq. \\
\hline 24 & 1499 & C. baccatum var. pendulum \\
\hline 25 & 1500 & C. baccatum var. pendulum \\
\hline 26 & 1501 & C. baccatum var. pendulum \\
\hline 27 & 1502 & C. annuum var. annuum \\
\hline 28 & 1503 & C. annuum var. annuum \\
\hline 29 & 1504 & C. baccatum var. pendulum \\
\hline 30 & 1551 & C. chinense Jacq. \\
\hline 31 & 1552 & C. chinense Jacq. \\
\hline 32 & 1553 & C. chinense Jacq. \\
\hline 33 & 1554 & C. chinense Jacq. \\
\hline 34 & 1555 & C. chinense Jacq. \\
\hline 35 & 1556 & C. baccatum var. pendulum \\
\hline 36 & 1557 & C. frutescens $\mathrm{L}$. \\
\hline 37 & 1558 & C. chinense Jacq. \\
\hline 38 & 1559 & C. annum var. glabriusculum \\
\hline 39 & 1560 & C. frutescens $\mathrm{L}$. \\
\hline 40 & 1561 & C. frutescens $\mathrm{L}$. \\
\hline 41 & 1562 & C. annuum var. annuum \\
\hline 42 & 1565 & C. annuum var. annuum \\
\hline 43 & 1566 & C. annuum var. annuum \\
\hline 44 & 1567 & C. annuum var. annuum \\
\hline 45 & 1568 & C. annuum var. annuum \\
\hline 46 & 1569 & C. annuum var. annuum \\
\hline 47 & 1570 & C. chinense Jacq. \\
\hline 48 & 1571 & C. chinense Jacq. \\
\hline 49 & 1572 & C. chinense Jacq. \\
\hline 50 & 1573 & C. baccatum var. pendulum \\
\hline 51 & 1574 & C. pubescens \\
\hline 52 & 1575 & C. annuum var. annuum \\
\hline 53 & 1576 & C. annum var. glabriusculum \\
\hline 54 & 1577 & C. chinense Jacq. \\
\hline 55 & 1578 & C. annuum var. annuum \\
\hline 56 & 1584 & C. baccatum var. baccatum \\
\hline 57 & 1585 & C. chinense Jacq. \\
\hline 58 & 1586 & C. chinense Jacq. \\
\hline 59 & 1587 & C. frutescens L. \\
\hline 60 & 1588 & C. frutescens L. \\
\hline
\end{tabular}

As funções discriminantes foram assim obtidas:

$$
\begin{aligned}
& D_{1}(x)=\ln \left(\rho_{1}\right)+\left(x-\frac{1}{2} \mu_{1}\right)^{\prime} \omega^{-1} \mu_{1} ; \\
& D_{2}(x)=\ln \left(\rho_{2}\right)+\left(x-\frac{1}{2} \mu_{2}\right)^{\prime} \omega^{-1} \mu_{2} ; \\
& \vdots \\
& D_{7}(x)=\ln \left(\rho_{7}\right)+\left(x-\frac{1}{2} \mu_{7}\right)^{\prime} \omega^{-1} \mu_{7} ;
\end{aligned}
$$

Onde:

$\pi_{1}$ a $\pi_{7}$ populações 1 a 7 .

$\mu_{1}$ a $\mu_{7}=$ vetor de médias das 15 variáveis avaliadas em $\pi_{1}$ a $\pi_{7}$

$\omega_{1}$ a $\omega_{7}=$ matriz de covariâncias entre $\mathrm{n}$ variáveis avaliadas em $\pi_{1}$ a $\pi_{7}$.

$\rho_{1}$ a $\rho_{7}=$ probabilidade, a priori, de os indivíduos pertencerem a $\pi_{1}$ a $\pi_{7}$.

$x=$ vetor de variáveis representativas dos caracteres envolvidos na análise.

Onde classificou-se o i-ésimo material genético com vetor de média $x_{\mathrm{i}}$ na população $\pi_{\mathrm{j}}$ se, e somente se, $D_{\mathrm{j}}\left(x_{\mathrm{i}}\right)$ foi o maior entre os elementos do conjunto $\left\{D_{1}\left(x_{\mathrm{i}}\right), D_{2}\left(x_{\mathrm{i}}\right), D_{3}\left(x_{\mathrm{i}}\right), D_{4}\left(x_{\mathrm{i}}\right), D_{5}\left(x_{\mathrm{i}}\right)\right.$, $D_{6}\left(x_{\mathrm{i}}\right)$ e $\left.D_{7}\left(x_{\mathrm{i}}\right)\right\}$.

A eficiência das funções discriminantes em classificar os genótipos, corretamente, nas populações previamente estabelecidas foi calculada pela taxa de erro aparente, que foi dada pela relação entre o número de classificações erradas e o número total de classificações (Cruz, 2001).

\section{RESULTADOS E DISCUSSÃO}

Oito grupos foram formados pelo método de Otimização de Tocher. O grupo I foi formado apenas por acessos da espécie $C$. anпиит var. annuит, reunindo $94 \%$ dos acessos avaliados dessa espécie. Apenas o acesso 10 (Tabela 1) de C. annuиm var. annuиm ficou no grupo II. Esse acesso diferenciou dos demais por ter cinco características divergentes, dentre elas, cor do caule, cor da folha cotiledonar, número de lóculos, hábito de crescimento e cor do fruto imaturo.

Além do acesso 10, o grupo II foi composto por todos acessos de $C$. frutescens $(12 ; 16 ; 36 ; 39 ; 40$ e 59$)$ e pelos acessos de C. baccatum. No grupo III mantiveram-se apenas acessos de C. chinense. O grupo IV foi composto pelos acessos 60 de C. frutescens e 49 de C. chinense, essas espécies são mui- 
to próximas, consideradas por alguns botânicos como uma única espécie (Heiser Júnior, 1976). Os acessos de $C$. annuum var. glabriusculum fazem parte do grupo V. Os acessos 48, 57 e 58, pertencentes à espécie $C$. chinense, formaram o grupo VI. No grupo VII, por sua vez, foram alocados os acessos $37 \mathrm{e}$ 47, de C. chinense.

O único acesso da espécie $C$. pubescens, acesso 51, formou o grupo VIII. Este grupo foi o mais distante, ficando totalmente isolado dos demais, sendo esta espécie a única não nativa do Brasil e pertencente ao complexo de flores roxas. Já as demais apresentam flores brancas.

Por meio do Método do VMP (Figura 1), pode-se inferir que existe divergência genética entre os 59 acessos estudados, devido à formação de 16 grupos distintos, considerando-se o corte no eixo x a 70\% de distância relativa entre os acessos. O grupo I conteve apenas acessos de C. annuum var. annuum. O grupo II foi composto por dois acessos de $C$. annuum var. annuum com características ornamentais, frutos pequenos com diversas cores. O grupo III pode ser subdividido em quatro sub-grupos (IIIa, IIIb, IIIc e IIId), considerando-se que o subgrupo IIIa reuniu todos acessos de C. frutescens do tipo 'Malagueta'; o sub-grupo IIIb foi composto apenas por acessos de C. baccatum var. baccatum e C. baccatum var. pendulum, e os sub-grupos IIIc e IIId foram compostos por apenas um acesso de $C$. chinense, cada um. No grupo IV foram alocados três acessos de $C$. chinense com cor do fruto laranja pálido e com formato campanulado. A maioria dos acessos estudados no presente trabalho teve frutos com formato redondo e pequenos. $\mathrm{O}$ grupo $\mathrm{V}$ foi representado apenas pelo acesso 49 da espécie $C$. chinense. No grupo VI ficou alocado o único acesso de $C$. frutescens do tipo 'Tabasco' com características distintas do tipo 'Malagueta'. Segundo Andrews (1995), as características tamanho, forma e posição do fruto são importantes para a identificação de cultivares, não sendo mais utilizadas em nível de espécie devido a grande pressão de seleção em relação a características do fruto. $\mathrm{O}$ grupo VII conteve oito acessos de $C$.

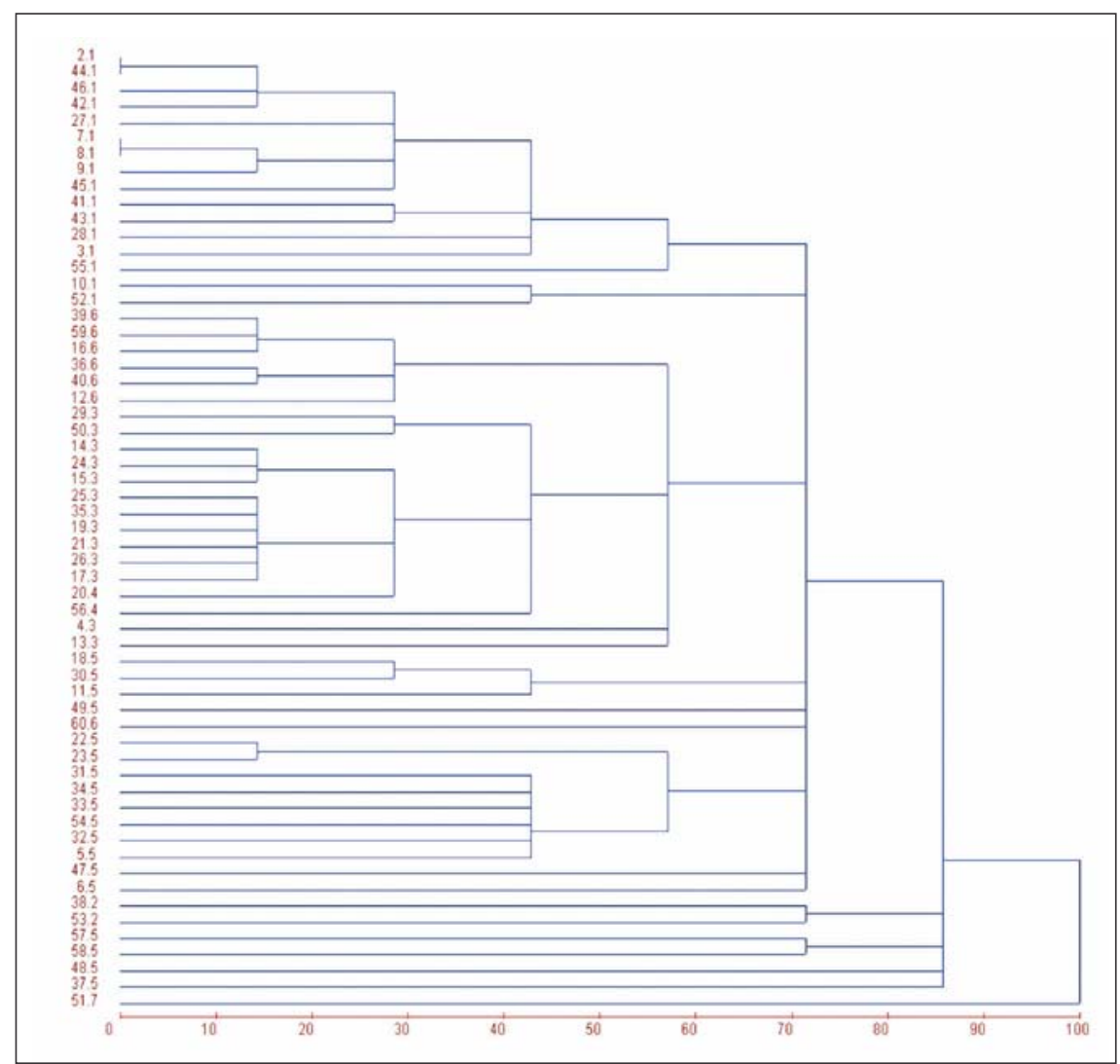

Figura 1. Dendrograma de dissimilaridades genéticas entre 59 acessos de Capsicum spp., obtido pelo método hierárquico do Vizinho Mais Próximo, com base na matriz de dissimilaridade com dados multicategóricos. No eixo x encontram-se as distâncias genéticas relativas e no eixo y encontra-se a descrição dos acessos, usando numeração do código do acesso seguido do número da espécie separado por ponto. Campos dos Goytacazes, UENF, 2002.

chinense de flores branco esverdeadas e frutos redondos. Os grupos VIII e IX foram formados por um acesso de $C$. chinense, cada. Os grupos X e XI foram formados pelos acessos de $C$. annuum var. glabriusculum. A espécie C. pubescens, pertencente ao grupo XVI divergiu completamente das demais, concordando com o Método de Tocher. Houve uma separação do grupo de flores brancas (do grupo I ao grupo IX) em relação ao grupo de flores roxas (do grupo X ao grupo XVI).

Para facilitar a visualização das espécies no dendrograma obtido pelo VMP, no eixo Y colocou-se ao lado do número do acesso o número correspondente da espécie botânica (1- C.annuum var. annuum; 2- C. annuum var. glabriusculum; 3- C. baccatum var. baccatum; 4- C. baccatum var. pendulum; 5- C. chinense; 6- C. frutescens, e 7-C. pubescens).

Com a projeção das distâncias no plano, sete grupos foram formados (Fi- gura 2). $\mathrm{O}$ grupo 1 reuniu exclusivamente acessos da espécie $C$. annuum var. annuum. Porém, dois acessos desta variedade botânica ficaram agrupados junto com os acessos de C. baccatum var. pendulum. $\mathrm{O}$ grupo 2 reuniu os acessos de $C$. annuum var. glabriusculum e ficou distante do grupo 1. O grupo 3 ( $C$. baccatum var. pendulum) englobou dois acessos que deveriam estar no grupo 1 e um acesso que deveria estar no grupo 6. O grupo 4 formou um grupo separado que ficou entre os grupos 3 e 5. Teoricamente esse grupo deveria estar mais próximo do grupo 3 , uma vez que pertence a mesma espécie diferindo apenas na variedade botânica. O grupo 5 (C. chinense), o maior grupo, ficou bem disperso no gráfico, provavelmente devido à grande variabilidade. É possível visualizar cinco subgrupos, confirmando os métodos já apresentados. Os acessos do grupo 6 estão bem próximos em ambos os eixos (x e y), porém, há uma interseção com o grupo 3 . O acesso da 
Tabela 2. Coeficientes de ponderação (constantes) obtidos para as funções discriminantes associados aos descritores que diferenciam as espécies de Capsicum. Campos dos Goytacazes, UENF, 2002.

\begin{tabular}{ccccccccccccccccc}
\hline $\begin{array}{c}\text { Função } \\
\left(\Psi_{\mathrm{i}}\right)\end{array}$ & $\begin{array}{c}\text { Constante } \\
\left(\mathbf{c}_{\mathbf{i}}\right)\end{array}$ & $X_{1}$ & $X_{2}$ & $X_{3}$ & $X_{4}$ & $X_{5}$ & $X_{6}$ & $X_{7}$ & $X_{8}$ & $X_{9}$ & $X_{10}$ & $X_{11}$ & $X_{12}$ & $X_{13}$ & $X_{14}$ & $X_{15}$ \\
\hline$\Psi_{1}$ & $-134,30$ & $-5,38$ & 6,88 & $-0,97$ & 2,03 & 15,48 & 14,79 & $-4,53$ & 2,92 & 1,71 & 2,11 & 24,01 & 10,79 & $-1,12$ & $-3,97$ & 7,76 \\
$\Psi_{2}$ & $-140,84$ & $-4,14$ & 8,62 & 0,66 & 0,47 & 16,95 & 13,83 & $-4,54$ & 2,51 & 1,93 & 1,15 & 18,17 & 10,24 & $-1,52$ & $-10,14$ & 8,21 \\
$\Psi_{3}$ & $-127,70$ & $-5,59$ & 4,94 & $-0,26$ & 1,04 & 15,59 & 14,42 & $-4,52$ & 2,84 & 1,69 & 2,35 & 24,67 & 11,15 & $-1,56$ & $-1,71$ & 8,38 \\
$\Psi_{4}$ & $-151,23$ & $-5,53$ & 4,76 & $-0,79$ & 4,78 & 15,73 & 15,18 & $-4,51$ & 3,17 & 3,32 & 4,00 & 23,04 & 11,94 & $-1,26$ & $-6,10$ & 7,33 \\
$\Psi_{5}$ & $-135,56$ & $-5,76$ & 6,45 & $-0,37$ & 2,12 & 15,66 & 14,62 & $-4,63$ & 2,82 & 1,72 & 2,25 & 24,22 & 11,10 & $-1,35$ & $-0,49$ & 7,73 \\
$\Psi_{6}$ & $-132,15$ & $-6,23$ & 6,26 & 0,23 & 2,85 & 15,54 & 14,62 & $-4,65$ & 2,49 & 1,19 & 3,54 & 23,94 & 10,77 & $-1,24$ & $-6,66$ & 5,38 \\
$\Psi_{7}$ & $-191,09$ & $-5,61$ & 6,22 & $-0,42$ & 1,98 & 15,63 & 14,62 & $-4,57$ & 2,81 & 1,71 & 2,50 & 53,48 & 10,99 & $-1,33$ & $-3,00$ & 7,60 \\
\hline
\end{tabular}

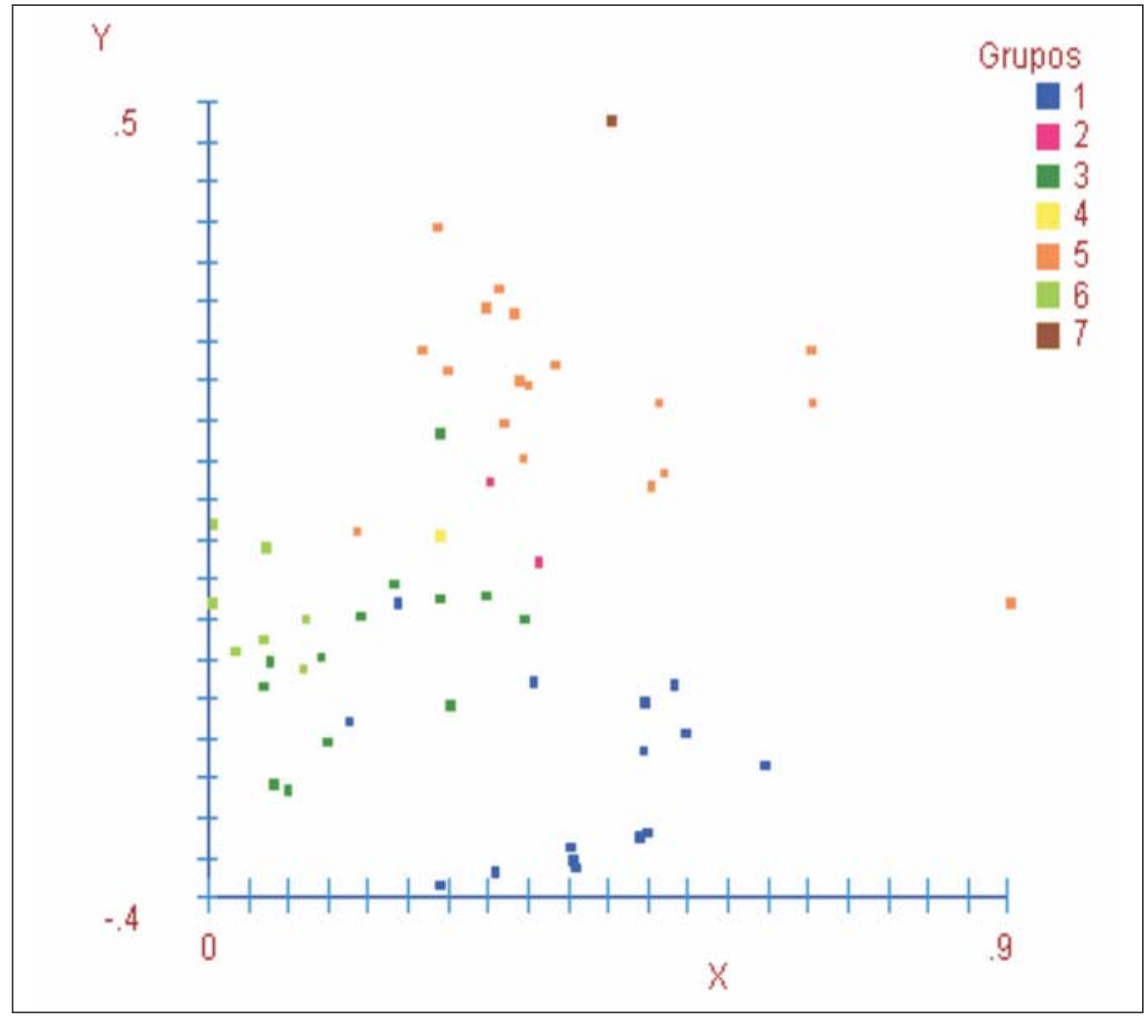

Figura 2. Projeção das distâncias no plano, considerando 59 acessos de Capsicum spp. Os grupos 1,2,3,4,5,6 e 7 referem-se as espécies ((1- C. апnиum var. annuит; 2- C. anпuит var. glabriusculum; 3- C. baccatum var. baccatum; 4- C. baccatum var. pendulum; 5- C. chinense; 6- C. frutescens, e 7- C. pubescens). Campos dos Goytacazes, UENF, 2002.

espécie $C$. pubescens formou um grupo bem isolado, concordando com os métodos de Tocher e VMP.

A Análise Discriminante de Anderson (1958) permitiu a obtenção de sete funções, descritas a seguir:

$$
\psi_{i}=c_{i}+\sum_{j=1}^{15} a_{i j} x_{j}
$$

$\Psi_{\mathrm{i}}=$ função discriminante associada à i-ésima espécie;

$c_{\mathrm{i}}=$ constante associada à i-ésima função discriminante; $a_{\mathrm{ij}}=$ coeficiente de ponderação do jésimo descritor na i-ésima função discriminante; e

$x_{\mathrm{j}}=$ valor do j-ésimo descritor.

Foram obtidos os valores conforme Tabela 2. A taxa de erro aparente foi de zero, demonstrando que as funções discriminaram $100 \%$ das espécies, e suas respectivas variedades botânicas em 59 acessos do gênero Capsicum spp. Portanto, o uso das variáveis multicategóricas confirma a importância dos descritores qualitativos denominados essenciais pelo IPGRI (1995) e permite caracterização e manejo eficientes de germoplasmas de Capsicum spp., mesmo quando se dispõem de poucos recursos financeiros.

Costa (2004), utilizando marcadores RAPD para estudar a divergência genética entre 58 dos 59 acessos testados no presente trabalho, constatou que não foi possível separar C. annuиm var. anпuиm de $C$. annuum var. glabriusculum e $C$. baccatum var. baccatum de C. baccatum var. pendulum. Todavia, essa distinção entre variedades botânicas foi possível utilizando-se os dados morfoagronômicos qualitativos e análise multivariada conduzidas no presente trabalho.

A coleta de dados multicategóricos é prática, econômica e demanda menor tempo comparado a dados quantitativos e dados moleculares. Porém, cada um tem sua importância singular, sendo preferível que uma coleção de germoplasma seja o mais amplamente estudada para dar maior suporte a pesquisas e ao banco de dados da coleção. A caracterização multicategórica e o estudo da divergência baseada nesses dados são alternativas viáveis para se estudar bancos e coleções de germoplasma que têm poucos recursos humanos e financeiros.

Concluiu-se, portanto, nas condições desse trabalho que características qualitativas analisadas pelo método de Tocher foram capazes de diferenciar as espécies de C.annuum var. annuum, $C$. annuum var. glabriusculum, $C$. chinense e C. pubescens. Ficaram no mesmo grupo acessos de $C$. frutescens e $C$. baccatum var. pendulum e C. baccatum var. baccatum.

O método de agrupamento de Tocher, o método hierárquico do Vizi- 
nho Mais Próximo e a Projeção das Distâncias no Plano foram concordantes e eficazes em agrupar materiais genéticos com pequena distância genética entre si.

Os 13 descritores qualitativos indicados pelo IPGRI (1995) como essenciais para caracterização morfoagronômica de Capsicum spp. devem ser utilizados no manejo de coleções e bancos de germoplasma.

A análise de variáveis multicategóricas demonstrou ter um grande potencial de uso na caracterização e manejo de bancos de germoplasma. As funções discriminantes de Anderson identificaram $100 \%$ das espécies e variedades botânicas estudadas.

\section{LITERATURA CITADA}

ABREU FB. 2001. Análise multivariada na determinação da divergência genética em feijão-devagem (Phaseolus vulgaris L.) de crescimento indeterminado. Campos dos Goytacazes: UENF, $73 \mathrm{f}$. (Tese de mestrado).
ABREU FB; MARIM BG; SILVA DJH; GUIMARÃES MA; LUCA CAC; FAGUNDES RAR. 2002. Determinação da divergência genética entre acessos de tomateiro mediante a utilização de variáveis multicategóricas. Horticultura Brasilei$r a, 20: 2$, Suplemento 2 .

ANDERSON TW. 1958. An introduction to multivariate statistical analysis. New York: John Wiley \& Sons, $345 \mathrm{p}$.

ANDREWS J. 1995. Pepper: The Capsicums domesticated. Texas: University of. North Texas. ASSIS GML. 2001. Análise Discriminante e Divergência Genética em espécies de Brachiaria. UFV: Viçosa, 76 f. (Dissertação de Mestrado). COIMBRA RR; MIRANDA GV; MOREIRA GR; SILVA DJH; CRUZ CD; CARNEIRO PCS; SOUZA LV; GUIMARÃES LJM; MARCASSO RC; CANIATO FF. 2001. Divergência genética de cultivares de milho baseada em descritores qualitativos. III SIRGEALC, p. 266-268.

COSTA FR. 2004. Diversidade genética entre acessos de Capsicum spp. com base em marcadores RAPD. UENF: Campos dos Goytacazes, 112 f. (Tese de mestrado).

CRUZ CD. 2001. Aplicativo computacional em genética e estatística. Viçosa: UFV. 648 p.

CRUZ CD; CARNEIRO PCS. 2003. Modelos biométricos aplicados ao melhoramento genético. Viçosa: UFV. 585 p.
FILGUEIRA FAR. 2000. Novo manual de olericultura: agrotecnologia moderna na produção e comercialização de hortaliças. Viçosa: UFV. $402 \mathrm{p}$.

HEISER JÚNIOR CB; 1976. Peppers. Capsicum (Solanaceae). In: Evolution of Crop Plants. Simmonds, N.W. (Ed.) Longman Scientific and Technical. p. 265-268.

IPGRI. 1995. Descriptores para Capsicum (Capsicum spp.). Roma: IPGRI. 51 p.

LIBERATO JÚNIOR. 1995. Aplicações técnicas de análise multivariada em fitopatologia. Viçosa: UFV. 144 f. (Tese de mestrado).

SUDRÉ CP. 2003. Divergência genética e avaliação da resistência à mancha bacteriana em Capsicum spp. UENF: Campos dos Goytacazes, $112 \mathrm{f}$. (Tese de mestrado).

SUDRÉ CP; RODRIGUES R; RIVA EM; KARASAWA M; AMARAL JÚNIOR AT. 2005. Divergência genética entre acessos de pimenta e pimentão utilizando técnicas multivariadas. Horticultura Brasileira, 23: 22-27. 